\title{
COMPARAÇÃO DAS FRAÇÕES HDL E LDL COLESTEROL COMO FATORES DE RISCO PARA A ATEROSCLEROSE CAROTÍDEA
}

\author{
RUBENS JOSÉ GAGLIARDI*, MARSAL SANCHES**, \\ ZIED RASSLAN"**, MARILDA LAURETTI S. GUEDES ${ }^{\star \star \star \star}$.
}

\begin{abstract}
RESUMO - Estudo prospectivo com o objetivo de verificar qual das frações do colesterol sanguíneo, HDL ou LDL, é mais significativa para o desenvolvimento da aterosclerose das artérias carótidas (AC). Constou de 125 indivíduos de ambos os sexos, com idade entre 45 a 75 anos, incluídos aleatoriamente. Em todos foi procedida a dosagem sanguínea do colesterol total $c$ das frações HDL e LDL e estudo ultrassonográfico (através de ultrassonografia modo B) das AC. Os resultados mostraram que a presença de placa de ateroma, com ou sem estenose nas AC , é inversamente proporcional aos níveis de HDL. Estes achados foram estatisticamente significantes ( $\times 2=6,57$ e $\times 2=9,24$, respectivamente para placa sem estenose e com estenose; $\times 2$ crítico $=5,99$ para $\alpha=5 \%$ ). Foi também constatada associação entre a presença de placa aterosclerótica na $A C$ e níveis sanguíneos elevados de LDL: quanto maiores os níveis de LDL, maior a proporção de indivíduos com placas, porém este achado não foi estatisticamente significante ( $x 2=0,97, x 2$ crítico 5,99 para $\alpha=5 \%)$. Não foi encontrada nesta casuística relação entre níveis elevados de LDL e presença de estenose nas AC. Estes resultados, ainda que iniciais, sugerem que níveis baixos de HDL sāo mais aterogênicos para as AC comparativamente a níveis elevados de LDL.
\end{abstract}

PALAVRAS CHAVE : aterosclerose, estenose, colesterol, artéria carótida.

\section{Comparative study of HDL and LDL cholesterol as risk factors for carotid atherosclerosis}

SUMMARY - In order to find out whether there exists a relationship between HDL and LDL serum levels and atherosclerotic plaques in the carotid artery (CA), a prospective study was carried out involving 125 patients. They were aleatorily included, of both sexes and age between 45-75 years old. HDL and LDL serum levels were measured as well CA ultrasonographic mode B investigated. It was verified that the number of patients having atherosclerotic plaques in CA was inversely proportional to the HDL levels. We came up with the same ratio when the HDL levels were compared to the number of patients having $C A$ stenosis. These results were statistically signifcant $(x 2=6.57$ and $\times 2=9.24$ respectively; critical $\times 2=5.99$ to $\alpha=5 \%)$. A direct ratio was also found out in the relationship between the presence of atherosclerotic plaques in the CA and the LDL serum levels (greater in patients with plaques). However no relationship between LDL levels and the occurrence or not of CA stenosis in patients having plaques was demonstrated. The results were not statistically significant ( $x 2=0.97$ and $\times 2=0.41$, respectively, critical $\times 2=5.99$ to $\alpha=5 \%$ ). The obtained results seem to be at least in part in agreement with literature findings. Statistically significative results in the comparison of HDL and LDL levels with the grade of stenosis of the CA were not found out ( $x 2=11.78$ and $x 2=4.03$, respectively; critical $\times 2=12.59$ to $\alpha=5 \%$ ).

KEY WORDS: atherosclerosis, stenosis, cholesterol, carotid artery.

A aterosclerose é enfermidade de desenvolvimento lento que acomete a túnica íntima das artérias musculares e elásticas médias e grandes ${ }^{16,17}$. Entre as teorias que explicam o processo

Estudo realizado na Disciplina de Neurologia (Liga da Aterosclerose) da Faculdade de Ciências Médicas da Santa Casa de São Paulo: * Professor Adjunto- Doutor, responsável pela Liga da Aterosclerose; ** Interno (6 ano); *** Professor Instrutor do Departamento de Clínica Médica; **** Professora Adjunta, chefe da Disciplina de Bioestatística (Departamento de Medicina Social).

Dr. Rubens José Gagliardi - Disciplina de Neurologia, Faculdade de Ciências Médicas da Santa Casa de São Paulo - Rua Cesário Motta Jr. 122 - 01222-001 São Paulo SP - Brasil. Fax 0018261288. 
aterosclerótico, tem grande aceitação a idéia de que o quadro sc origine de lesāo endotelial, em que interagem células sanguineas, principalmente monócitos, que fagocitam ésteres de colesterol e penetram na camada íntima da artéria, passando a constituir as células espumosas. Isto ocorrendo repetidamente leva ao aparecimento da estria gordurosa, que é a alteração inicial da aterosclerose. Posteriormente, células musculares lisas migram da túnica da camada média para a íntima; ocorre proliferaçāo de fibras musculares lisas e de fibroblastos, com produção de fibras elásticas colágenas. Todas estas alterações levam a espessamento excêntrico da parede da artéria e às vezes com redução da sua luz: é a placa aterosclerótica que pode evoluir com as suas temíveis complicaçōes. Além da estenose na luz da artéria, a placa pode apresentar outras complicações, como alterações degenerativas. A degeneração da placa leva ao surgimento de úlceras, fendas e fissuras em sua superfície, o que estimula processos de agregação e adesão de plaquetas e formação de trombose. A organização posterior acarreta aumento das dimensões da placa. A estenose pode levar a obstrução do vaso e a rotura da placa (ou os seus fragmentos) pode provocar embolias ${ }^{16,17}$. Achados experimentais mostraram que na aterosclerose, além do fator puramente mecânico de redução da luz, existe diminuição da capacidade de relaxamento vascular por comprometimento endotelial ${ }^{8}$.

Os fatores de risco para a aterosclerose são múltiplos e muitas vezes estão associados: dislipidemias, hipertensão arterial, tabagismo, sedentarismo, diabetes e outros secundários. A prevençāo c/ou controle de tais fatores têm aumentado consideravelmente a expectativa de vida, principalmente em países desenvolvidos, onde se realizam pesquisas epidemiológicas por estudos longitudinais e transversais, seguidos por programas de intervençăo $0^{11,16,19}$. As dislipidemias e, principalmente, as alterações do metabolismo das lipoproteínas sāo causas fundamentais das doenças ateroscleróticas ${ }^{9.16}$. As lipoproteínas são partículas globulares de alto peso molecular que transportam os triglicérides e o colesterol ingeridos diariamente. Existem cinco classes principais de lipoproteínas: quilomicrons, VLDL, IDL, LDL, HDL, diferindo uma da outra, segundo a composição da apoproteina, densidade, tamanho e mobilidade eletroforética ${ }^{16,17}$. Modernamente tem-se dado ênfase a duas alterações de lipoproteínas como importante fator de risco para a aterosclerose: o aumento dos níveis séricos das lipoproteínas de baixa densidade (LDL) e a diminuição dos níveis de lipoproteínas

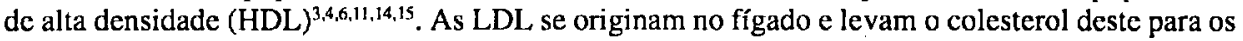
tecidos periféricos. Sua composição é de $45 \%$ de colesterol, $22 \%$ de fosfolípides, $21 \%$ de proteínas; o restante corresponde a triglicérides e ácidos graxos. $\mathrm{O}$ HDL consiste de quantidades quase iguais de lípides e proteínas. Os lípides compreendem ésteres de colesterol, colesterol livre, triglicérides e fosfolípides. As proteínas são basicamente as apoproteínas AI, A, C, E. Realizam o transporte reverso do colesterol dos tecidos periféricos para o fígado ${ }^{3}$. Vários métodos tem sido empregados para 0 diagnóstico de placas ateroscleróticas e de estenose e atualmente tem sido muito utilizada a ultrassonografia. É procedimento incruento, com bom índice de precisāo, bastante fiel e confiável. Atualmente é o método mais utilizado para esse estudo ${ }^{1,12}$.

Embora a associação entre baixos níveis de HDL, altos de LDL e aterosclerose coronária já tenha sido demonstrada por meio de diversos estudos, esta relação não está bem definida no que se refere ao acometimento das artérias carótidas $(A C)$, cerebrais e vertebrobasilares ${ }^{2,5,6,13,15,21}$. O presente estudo visa a verificar se há associação entre baixos níveis sanguíneos de HDL ou de altos níveis de LDL com o desenvolvimento de aterosclerose nas AC e qual das duas fraçōes seria a mais importante no desenvolvimento da doença. Tenta também correlacionar estas alteraçōes lipídicas com a presença ou não de placas de estenose.

\section{CASUÍSTICA E MÉTODOS}

Estudo prospectivo em 125 indivíduos, de ambos os sexos, com idade entre 45 a 75 anos acompanhados no ambulatório da "Liga da Aterosclerose" (Disciplina de Neurologia) da Santa Casa de São Paulo no período de março-1990 a julho-1992. Os indivíduos foram incluídos na casuística aleatoriamente, sendo a idade o único critério de exclusão.

Para efeito deste estudo, os indivíduos desta casuística (ao lado de cuidados clínicos e neurológicos usuais) foram submetidos a dois exames complementares básicos: dosagem sanguínea dos niveis das frações HDL e LDL colesterol; ultrassonografia modo B das AC. 
A dosagem dos níveis sanguíneos do LDLe do HDL colesterol foi realizada por método enzimático (colesterol oxidase/peroxidase) com leitura em espectofotômetro a $505 \mathrm{~mm}$, em sangue venoso, com jejum de 12 horas.

O exame ultrassonográfico foi realizado com o parelho Shimadzo, modelo SLD-300, com transductores conversos de 7,5 $\mathrm{mHz}$. Os doentes foram colocados em decúbito dorsal, usando-se um apoio na regiāo cervical posterior. Os exames foram realizados com cortes longitudinais da regiāo mediana em direção à face lateral do pescoço e cortes transversais da região próxima ao ramo inferior da mandíbula às regiōes supraclaviculares.

Neste estudo não foi valorizada a localizaçāo da placa, considerando-se genericamnete que as placas, quando presentes, se encontravam nas $\mathrm{AC}$.

Os indivíduos foram classificados segundo o nível do HDL e LDL, acompanhando os padrões clássicos de normalidade. Quanto ao $\mathrm{HDL}$ foram divididos em 3 grupos: menores do que $35 \mathrm{mg} / \mathrm{dL}$, entre $36 \mathrm{e} 55 \mathrm{mg} / \mathrm{dL}$. e maiores do que $55 \mathrm{mg} / \mathrm{dL}$. Conforme o nível sanguíneo do LDL, os indivíduos também foram divididos em 3 grupos: menores do que $120 \mathrm{mg} / \mathrm{dL}$, entre 121 a $160 \mathrm{mg} / \mathrm{dL}$ e maiores do que $160 \mathrm{mg} / \mathrm{dL}$. Os doentes com placas de aterosclerose nas AC foram divididos em 2 grupos: com placa sem estenose; com placa com estenose. Quanto ao grau de estenose, a casuística foi classificada em 4 subgrupos: sem estenose, estenose de 1 a $20 \%$, estenose de 21 a $70 \%$ e estenose superior a $70 \%$.

A análise estatística foi realizada pelo teste do qui-quadrado (x2), adotando-se intervalo de confiança de $95 \%(\alpha=5 \%)$

\section{RESULTADOS}

A Tabela 1 mostra a proporção de doentes com placa aterosclerótica nos diferentes subgrupos por níveis de HDL. A Tabela 2 mostra o mesmo em relação aos subgrupos do LDL.

Observa-se na Tabela 1 a nítida tendência da proporção de pacientes sem placa de ateroma nas AC aumentar à medida que aumentam os níveis de HDL. Inversamente verifica-se que há tendência a diminuição da proporção de indivíduos com placa quando os níveis de HDL diminuem. Estas

Tabela 1. Relação entre os níveis sanguíneos de HDL e a incidência de placa aterosclerótica nas AC.

\begin{tabular}{ccccccc}
\hline níveis sanguíneos de HDL (mg/dL) & \multicolumn{2}{c}{ sem placa } & \multicolumn{2}{c}{ com placa } & \multicolumn{2}{c}{ total } \\
& $\mathrm{N}$ & $\%$ & $\mathrm{~N}$ & $\%$ & $\mathrm{~N}$ & $\%$ \\
\hline \hline 35 & 6 & 21,4 & 22 & 78,6 & 28 & 100 \\
$35-55$ & 23 & 33,0 & 48 & 67,0 & 71 & 100 \\
$>55$ & 14 & 34,4 & 12 & 65,6 & 26 & 100 \\
\hline
\end{tabular}

$x 2=6,57 \times 2$ critico $(\alpha=5 \%)=5,99$. Estatisticamente significante.

Tabela 2. Relação entre os niveis sanguineos de $L D L$ e a incidência de placa aterosclerótica nas $A C$.

\begin{tabular}{cccccccc}
\hline níveis sanguíneos de LDL (mg dL) & \multicolumn{2}{c}{ sem placa } & \multicolumn{2}{c}{ com placa } & \multicolumn{2}{c}{ total } \\
& $\mathrm{N}$ & $\%$ & $\mathrm{~N}$ & $\%$ & $\mathrm{~N}$ & $\%$ \\
\hline$<120$ & 13 & 38,2 & 21 & 61,8 & 34 & 100 \\
$121-160$ & 18 & 36,7 & 31 & 63,3 & 49 & 100 \\
$>160$ & 12 & 28,6 & 30 & 71,4 & 42 & 100 \\
\hline
\end{tabular}


Tabela 3. Relaçäo entre os niveis sanguíneos de HDL e a incidência de estenose nas $A C$.

\begin{tabular}{ccccccc}
\hline niveis sanguíneos de HDL (mg/dL) & \multicolumn{2}{c}{ sem estenose } & \multicolumn{2}{c}{ com estenose } & \multicolumn{2}{c}{ total } \\
& $\mathrm{N}$ & $\%$ & $\mathrm{~N}$ & $\%$ & $\mathrm{~N}$ & $\%$ \\
\hline$<35$ & 6 & 27,3 & 16 & 72,7 & 22 & 100 \\
$36-55$ & 29 & 60,5 & 19 & 39,5 & 48 & 100 \\
$>55$ & 9 & 75,0 & 3 & 25,0 & 12 & 100 \\
\hline
\end{tabular}

$x 2=9,24 \times 2$ critico $(\alpha=5 \%)=5,99$. Estatisticamente significante.

Tabela 4. Relação entre os níveis sanguíneos de LDL e a incidência de estenose nas $A C$.

\begin{tabular}{ccccccc}
\hline níveis sanguíneos de LDL (mg/dL) & \multicolumn{2}{c}{ sem estenose } & \multicolumn{2}{c}{ com estenose } & \multicolumn{2}{c}{ total } \\
& $\mathrm{N}$ & $\%$ & $\mathrm{~N}$ & $\%$ & $\mathrm{~N}$ & $\%$ \\
\hline \hline 120 & 11 & 52,3 & 10 & 47,7 & 21 & 100 \\
$121-160$ & 18 & 58,0 & 13 & 42,0 & 31 & 100 \\
$>160$ & 15 & 50,0 & 15 & 50,0 & 30 & 100 \\
\hline
\end{tabular}

$x 2=0,41 \times 2$ critico $(\alpha=5 \%)=5,99$. Não significante estatisticamente.

Tabela S. Relação entre os niveis sanguíneos de HDL e o grau de estenose nas $A C$.

\begin{tabular}{|c|c|c|c|c|c|c|c|c|c|c|}
\hline \multirow{3}{*}{ níveis de HDL (mg/dL) } & \multicolumn{10}{|c|}{ Grau de Estenose } \\
\hline & \multicolumn{2}{|c|}{ zero } & \multicolumn{2}{|c|}{1 a $20 \%$} & \multicolumn{2}{|c|}{21 a $70 \%$} & \multicolumn{2}{|c|}{$>70 \%$} & \multicolumn{2}{|c|}{ total } \\
\hline & $\mathbf{N}$ & \% & $\mathbf{N}$ & $\%$ & $\mathbf{N}$ & $\%$ & $\mathbf{N}$ & $\%$ & $\mathbf{N}$ & $\%$ \\
\hline$<35$ & 6 & 27,3 & 5 & 22,7 & 6 & 27,3 & 5 & 22,7 & 22 & 100 \\
\hline $36-55$ & 29 & 60,5 & 4 & 8,3 & 11 & 22,9 & 4 & 8,3 & 48 & 100 \\
\hline$>55$ & 9 & 75,0 & - & - & 2 & 16,7 & 1 & 8,3 & 12 & 100 \\
\hline
\end{tabular}

$x 2=11,78 \times 2$ crítico $(\alpha=5 \%)=12,59$. Não significante estatisticamente.

variaçōes sāo estatisticamente significantes $(\times 2=6,57 ; \times 2$ crítico=5,99 com intervalo de confiança de 95\%). A Tabela 2 mostra tendência à diminuição da proporçāo de indivíduos sem placa à medida que os niveis de LDL aumentam, bem como há tendência a aumento da proporção de indivíduos com placa à medida que os níveis de LDL aumentam. Estas variaçōes observadas na Tabela 2 nāo são tão evidentes quanto as constatadas na Tabela 1 e não são estatisticamente significantes $(x 2=0,97$; x 2 crítico=5,99 para intervalo de confiança de $95 \%$ ).

A Tabela 3 mostra a relação entre os níveis de HDL e a incidência de estenose nas AC. A Tabela 4 mostra a relação entre LDL e incidência de estenose.

Verifica-se que a proporção de indivíduos sem estenose aumenta à medida que aumentam os níveis de HDL, ao mesmo tempo que ocorre decréscimo da porcentagem de doentes com estenose na AC. Trata-se de uma diferença estatisticamente significante $(x 2=9,24 ; \times 2$ crítico=5,99 com intervalo de confiança de $95 \%$ ). Conforme se nota pela Tabela 4 , não se evidenciou relação nítida entre os níveis de LDL e a presença de placas atereroscleróticas nas $\mathrm{AC}$ e estes resultados não são estatisticamente significantes ( $\times 2=0,41 ; \times 2$ crítico $=5,99$ para intervalo de confiança de $95 \%$ ). 
Tabela 6. Relaçāo entre us niveis sanguineos de LDL e o grau de estenose das $A C$.

Grau de Estenose

\begin{tabular}{ccccccccccc} 
níveis de LDL (mg/dL) & \multicolumn{2}{c}{ zero } & \multicolumn{2}{c}{1 a $20 \%$} & \multicolumn{2}{c}{21 a $70 \%$} & \multicolumn{2}{c}{$>70 \%$} & \multicolumn{2}{c}{ total } \\
& $\mathrm{N}$ & $\%$ & $\mathrm{~N}$ & $\%$ & $\mathrm{~N}$ & $\%$ & $\mathrm{~N}$ & $\%$ & $\mathrm{~N}$ & $\%$ \\
\hline$<120$ & 11 & 52,4 & $\mathrm{I}$ & 4,8 & 5 & 23,8 & 4 & 19,0 & 21 & 100 \\
$121-160$ & 18 & 58,1 & 4 & 12,9 & 5 & 16,1 & 4 & 12,9 & 31 & 100 \\
$>160$ & 15 & 50,0 & 4 & 13,3 & 9 & 30,0 & 2 & 6,7 & 30 & 100 \\
\hline
\end{tabular}

$x 2=4,03 \times 2$ critico $(\alpha=5 \%)=12,59$. Não significante estatisticamente.

As Tabelas 5 e 6 mostram a relação entre os niveis de HDL e LDL com o grau de estenose das AC.

À medida que os níveis de HDL aumentam, verifica-se que a proporção de indivíduos sem estenose também aumenta; a proporção de indivíduos com estenose de 1 a $20 \%$ e de 21 a $70 \%$ diminui e a proporção dos indivíduos com estenose acima de $70 \%$ tende a diminuir. Estes resultados não são estatisticamente significantes $(\times 2=11,78 ; \times 2$ crítico=12,59 para intervalo de confiança de 95\%). Constata-se, pela Tabela 6 , que à medida que aumentam os niveis sanguíneos de LDL a porcentagem de indivíduos sem estenose inicialmente se eleva (até os níveis médios de $160 \mathrm{mg} / \mathrm{dL}$ ) e diminuem com as taxas médias superiores a $160 \mathrm{mg} / \mathrm{dL}$. Com a elevação das taxas de LDL, a proporção de doentes com estenose entre 1 a $20 \%$ tende a se elevar; a proporção de doentes com estenose entre 21 e $70 \%$ sofre inicialmente queda, e posteriormente, elevação; e existe tendência a diminuir a proporção dos doentes com estenose superior a $70 \%$. Estes resultados nao são estatisticamente significantes ( $\mathrm{x} 2=4,03 ; \mathrm{x} 2$ crítico=12,59 com intervalo de confiança de $95 \%$ ).

\section{COMENTÁRIOS}

As doenças isquêmicas cerebrovasculares sāo importante causa de óbito e incapacitações no Brasil ${ }^{10}$ e a constatação de parâmetros seguros para a avaliação de seus fatores de risco constitui o primeiro passo para o planejamento e estabelecimento de medidas preventivas. A relaçāo entre os níveis de HDL e LDL tem sido, modernamente, considerado bom parâmetro prático para a avaliação do risco de doenças cardiovasculares isquêmicas. Numerosos estudos indicam que o risco da aterosclerose coronariana é inversamente proporcional aos níveis séricos de HDL e diretamente aos do LDL ${ }^{3,4,6,15}$.

Em relação a aterosclerose das AC e a ação das frações HDL e LDL há escassez de estudos. Estes dados ainda nāo estão suficientemente esclarecidos, como ocorre quanto ao comprometimento das coronárias. Airo e col. ${ }^{2}$, $\mathrm{em}$ amplo estudo desenvolvido na Itália, constataram que os níveis de HDL nāo estavam significativamente reduzidos em doentes com lesăo isquêmica cerebrovascular. Ao contrário, Murai e col. ${ }^{13}$, verificaram que as taxas de HDL e a relação entre o HDL e o LDL constituem os únicos parâmetros significativamente alterados nos doentes com patologia cerebrovascular isquêmica. Estudos recentes ${ }^{9.18}$ reforçam este ponto de vista, que parece ser a tendência atual.

O presente estudo constatou que a proporção de indivíduos sem placa de ateroma nas AC aumentava a medida que os índices de HDL se elevavam, ao mesmo tempo em que a proporção de doentes com placa apresentava nítida tendência de queda (Tabela 1). Esta diferença é estatisticamente significante. Tais resultados, à semelhança da maioria dos relatos da literatura, permitem concluir que, em nossa casuística, taxas baixas de HDL sanguíneo, constituem fator de risco para o desenvolvimento de placa de ateroma nas AC. Estes resultados são aparentemente semelhantes ao de Murai e $\mathrm{col}^{13}$, porém deve-se frisar que o presente estudo analisou exclusivamente a presença de placa nas AC, independentemente da ocorrência ou não de doença cerebrovascular isquêmica, ao passo que no estudo citado foram incluídos apenas doentes que já tivessem sido vítima de alguma forma de doença cerebrovascular isquêmica. 
A associação entre os níveis sanguíneos de LDL e a presença de placa de ateroma nas AC também foi motivo de análise (Tabela 2). Observa-se discreta tendência a aumento na proporçāo de indivíduos com placa a medida que os índices de LDL aumentam, bem como tendência a diminuição da proporçāo de indivíduos sem placa. Neste subgrupo, entretanto, estes resultados não são significativos estatisticamente. $O$ fato de estes resultados não serem estatisticamente significantes, nāo permite conclusão definitiva, pois a amostra năo foi numerosa o suficiente para conclusões definitivas. Este achado é diferente dos relatos clássicos para a aterosclerose coronariana e necessita de novas avaliaçōes, com maior número de casos.

Nos indivíduos com placa de ateroma nas AC, a relação entre os níveis de HDL e a presença de estenose nas AC, também foi alvo de análise (Tabelas 3 e 4). Constata-se que à medida que aumentam os índices de HDL, a proporção de indivíduos sem estenose também aumenta e diminui a proporção de casos com estenose (Tabela 3). Estes resultados são estatisticamente significantes. É possível concluir, que nesta casuística, o risco de ocorrência de estenose em indivíduos com aterosclerose nas AC diminui à medida que as taxas de HDL se elevam. Na Tabela 4 se observa que não há associação entre os níveis de LDL e a presença ou não de estenose nas AC. Estes dados não devem ser tomados como conclusivos, pois o número da amostragem foi pequeno e não é possível se obter resultados definitivos a partir desta amostra. Novos estudos a este respeito estāo sendo feitos.

Outro item analisado foi o grau de estenose das $\mathrm{AC}$ e a possível relação com as frações HDL e LDL. Foi constatada discreta tendência a diminuição da proporçāo de doentes com estenose, nos diversos graus, à medida que os níveis de HDL se elevam (Tabela 5). Tais resultados nāo sāo estatisticamente significantes. $O$ fato de não serem estes resultados estatisticamente significantes, não necessariamente implica na conclusão de nāo haver associação entre os níveis de HDL e o grau de estenose consequente a placa, mas sim indica que o tamanho da amostra estudada não é suficiente para uma análise como esta. A mesma observação deve ser feita para os resultados apresentados na Tabela 6, em que nāo se verifica relação nítida ou linear entre os níveis de LDL e os diferentes graus de estenose das AC. Estes resultados não são estatisticamente significantes. Isto não indica que os resultados encontrados sejam falsos, mas sim que o tamanho da amostra não foi grande o suficiente para que estatisticamente, estes resultados pudessem ser considerados fidedignos.

Assim pode-se concluir que, em nossa casuística, existe relação inversa entre os níveis de HDL e a presença de placa aterosclerótica na $\mathrm{AC}$, de modo que a proporçāo de doentes com placa é inversamente proporcional aos níveis de HDL. Embora os resultados pareçam indicar que a proporção de doentes seja diretamente proporcional aos niveis de LDL, tais resultados nāo foram estatisticamente significantes, sendo necessário outros estudos, com amostras de dimensões maiores, a fim de verificar se tal relação realmente existe.

A análise dos grupos de doentes com estenose revelou que a proporção destes doentes tende a diminuir à medida que os niveis de HDL aumentam. Tais resultados săo estatisticamente significantes. Baseando-se nesta afirmação, pode-se notar que há tendência entre os doentes com taxas elevadas de HDL de menor incidência de aterosclerose e estenose na AC. O HDL colesterol se apresentou como fator de proteçāo para a aterosclerose da $\mathrm{AC}$ e, consequentemente, sua baixa concentração sanguínea ( $<35 \mathrm{mg} / \mathrm{dL}$ ) se mostra como fator de risco, independentemente da concentração do LDL.

Apresenta-se como bastante interessante a possibilidade de comprovaçāo de que taxas baixas de HDL possam ser fator de risco isolado para a aterosclerose carotídea. Novos estudos são necessários para avaliar se o HDL menor do que $35 \mathrm{mg} / \mathrm{dL}$ pode ser um fator de risco, independentemente da presença de outros fatores, a interrelação das dislipidemias com os demais fatores de risco para a aterosclerose e detalhar o comportamento de determinadas lipoproteínas, como a $L p(a)$ e a $L p(A 1)$, que têm sido, em publicaçōes recentes, fortemente implicadas no desenvolvimento da doença cerebrovascular aterosclerótica ${ }^{14,18,20,21}$. 


\section{REFERÊNCIAS}

1. Ackerman RH.,Candia M. Identifying clinically relevant carotid disease.Stroke 1994, 25:1-3.

2. Airo R, Ferrari CM. High density lipoproteins and apolipoprotein $\mathrm{A}$ in cerebrovascular desease. Atherosclerosis 1985, 54:343-348,

3. Brown WV. Lipoproteins: what, when and how often to measure. Heart Dis Stroke 1992, 1:20-26.

4. Fermer JA. Papel do HDL colesterol e dos triglicérides na prevenção da aterosclerose. Lipid Digest 1992, 1:4-7.

5. Frick MH, Elo O, Haapa K, Heinonen OP, Heinsalmi P, Hello P, Huttunen JK, Kaitaniemi P, Nikkilă EK. Helsinky heart study: primary prevention trial with gemfibrozil in middle age men with dyslipidimia. $\mathrm{N}$ Engl J Med 1987, 317:1237-1245.

6. Gordon T, Castelli WP, Hjortland MC, Kannel WB, Dawber T. High density lipoprotein as a protective factor against coronary heart disease: the Framingham Study. Am J Med 1977, 62:707-714.

7. Jürgens G, Költringer P. Lipoprotein (a) in ischemic cerebrovascular disease: a new approach to the assessment of risk for stroke. Neurology 1987, 37: 513-515.

8. Kanamaru K, Waga S, Tochio $H$, Nagatani $K$. The effect of atherosclerosis on endothelium dependent relaxion in aorta and intracranial arteries of rabbits. J Neurosurg 1989, 70:795-798.

9. Konishi M, Iso H, Komachi Y, Iida M, Shimamoto T, Jacobs DR, Terao A, Baba S, Sankai T, Masashi I. Associations of serum total cholesterol, different types of stroke, and stenosis distribution of cerebral arteries: the Akita pathology study. Stroke 1993, 24:954-964.

10. Lessa I. As doenças cerebrovasculares e as isquêmicas do coração como causa de morte no Brasil. Medicina Prat 1990, 4:65-68.

11. Martins IS, Coelho LT, Matos MS, Mazzilli RN,Trigo M, Wilson D. Dislipidemias e alguns fatores de risco associados em uma população periférica da região metropolitana de São Paulo, SP, Brasil: um estudo piloto. Rev Saude Publ. São Paulo 1989, 23:236-243.

12. Mitt] RL, Broderick M, Carpenter JP, Goldbeg HI, Listerud J, Mishkin MM, Berkowitz HD, Atlas SW. Blinded-reader comparison of magnetic resonance angiography and duplex ultrasonography for carotid artery bifurcation stenosis. Stroke 1994, 25:4-10.

13. Murai A, Tanaka T, Miyahara T, Kameyama M. Lipoprotein anormalities in the pathogenesis of cerebral infarction and transient isquemic attack. Stroke 1981, 2:167-172.

14. Nagayama M, Shinohara Y, Nagayama T. Lipoprotein (a) and ischemic cerebrovascular disease in young adults. Stroke 1994, 25:74-78.

15. Ramires JAF. O perfil do HDL. Lipid Digest 1992, 1:2-11.

16. Ross R. Aterosclerose, In Wyngaarden JB, Smith LH, Bennett JC. Cecil tratado de medicina interna. Rio de Janeiro: Guanabara-Koogan. 1993, p.299-303.

17. Ross R. The pathogenesis of atherosclerosis: a perspective for the 1990s. Nature 1993, 362:801-809.

18. Shintani S, Kikuchi S, Hamaguchi H, Shiigai T. Higth serum lipoprotein (a) levels are an independent risk factor for cerebral infarction Stroke 1993, 24:965-969.

19. Thelle D. Women and coronary heart disease: a review with special emplasis on some risk factors. Lipid Rev 1990, 4:34-39.

20. Valentine RJ, Graybum PA, Vega GL, Grundy SM, Lp(a) lipoprotein is an independent, discriminating risk factor for premature peripheral atherosclerosis among white men. Arch Intern Med 1994,154:801-806.

21. Yatsu FM, Kasturi R, Alam R. Gene polymorphism of apolipoprotein Al: the major protein of hight density lipoprotein in predicting stroke risk among white and black subjects. Stroke 1993, 24:I 26-1 32. 